\title{
The 9th Congress on Alternatives and Animal Use in the Life Sciences
}

What a World Congress! More than a thousand participants from 49 countries convened at the Hilton Prague to exchange their ideas and insights over the course of five days this August. 891 abstracts had been accepted (http://www.altex.ch/ ALTEX-Proceedings.90 $\mathrm{html}$ ) for oral or poster presentations. Nine sessions ran in parallel throughout the Congress to discuss the multitude of themes and aspects pertaining to the use of animals in scientific experiments and the reduction, refinement and replacement of their use. The sessions were complemented by daily poster sessions and framed by insightful and thought provoking plenary lectures. The scientific program was complemented by an exceptional social program, allowing participants to experience the city and culture of Prague while catching up with colleagues.

Overall there has been major progress in the 3 Rs field. The "Mahatma-Gandhi-Doerenkamp Center for alternatives to the use of animals in life science education" had a spectacular announcement to make: Animal dissection has been banned in university education courses throughout India. This will save an estimated 30 million animals per year, including endangered species of frogs.

Refinement and animal welfare was a subject that was more prominent than at previous World Congresses. This is a dynamic and highly interesting area that sensitizes us to the needs and abilities of laboratory animals so that in the cases where animal experiments cannot yet be replaced, the animals may have a better quality of life, leading to more robust results.

For the first time, the publication, availability and application of 3Rs methods were given a broad stage and were received with much interest. Accessibility to databanks and journal articles, and proper reporting of experiments are key issues in avoiding replication of animal experiments and informing scientists on available alternatives. In a session on the role of journals in supporting the 3 Rs, editors from four journals, including ALTEX, discussed the role of open access publication, applica- tion of guidelines for reporting of animal experiments and the opportunities and challenges that "big data" hold for alternative methods.

A number of prestigious prizes were awarded at WC9: The Russell and Burch Award was presented by the Humane Society of the United States to Martin Stephens of CAAT. Dan Weary from the University of British Columbia received the Charles River Laboratories' Excellence in Refinement Award. FICAM director Tuula Heinonen received the Björn Ekwall Award and Miriam N. Jacobs was awarded the ALTEX Prize.

The social program started off with a get-together party at the congress venue, progressed to a welcome reception at the Public Spa on the bank of the Vltava and culminated in a superlative Art Nouveau gala dinner in the Prague Municipal House. These events gave everyone the opportunity to meet colleagues in person, catch up with old friends and to informally assess possibilities to cooperate on new projects.

In the closing ceremony all co-organizers and sponsors were thanked for their excellent contributions, especially the staff from Guarant International. In all, it was a fantastic congress, as also reflected by the photographs and the feedback from participants to the co-chairs Dagmar Jírová and Horst Spielmann posted on the website http://www.wc9prague.org/

The $10^{\text {th }}$ World Congress, co-chaired by Elaine Faustman, Robert Kavlock and Joanne Zurlo under the motto "The 3Rs in Action" will take place in Seattle in September 2017. There we will come together again to determine where alternative methods stand. What approaches were successful and what has been achieved? What was not - and why? What needs to be changed? What subjects are pressing and how can new technologies help? There will be much to discuss in 2017. The dynamics and the rapid developments in the $3 \mathrm{Rs}$ area give us hope that the vision of a world without animal experiments may indeed become a reality.

Stefanie Schindler and Sonja von Aulock 